\title{
Triple Helix and Random Coil of Scleroglucan in Dilute Solution
}

\author{
Toshio YANAKI and Takashi NORISUYE \\ Department of Macromolecular Science, Osaka University, \\ Toyonaka, Osaka 560, Japan \\ (Received February 18, 1983)
}

\begin{abstract}
Eleven sonicated samples of a polysaccharide scleroglucan (chemically identical with but different in biological origin from schizophyllan) in $0.01 N$ sodium hydroxide $(\mathrm{NaOH})$ and dimethylsulfoxide (DMSO) at $25^{\circ} \mathrm{C}$ were studied by light scattering, viscometry, and ultracentrifugation. Data for the radius of gyration $\left\langle S^{2}\right\rangle^{1 / 2}$, the intrinsic viscosity [ $\left.\eta\right]$, and the sedimentation coefficient $s_{0}$ as functions of weight-average molecular weight $M_{w}$, combined with those for the ratio $M_{w}(0.01 N \mathrm{NaOH}) / M_{w}$ (DMSO), showed that scleroglucan dissolves in DMSO as a single randomly coiled chain very similarly to that of schizophyllan in the same solvent, while it dissolves in $0.01 \mathrm{~N} \mathrm{NaOH}$ as a rodlike trimer or higher aggregates, depending on whether $M_{w}$ (DMSO) is lower or higher than $2 \times 10^{5}$. From $\left\langle S^{2}\right\rangle$, [n], and $s_{0}$ for samples with $M_{w}$ (DMSO) $\lesssim 1 \times 10^{5}$ in $0.01 \mathrm{~N} \mathrm{NaOH}$, the contour length per main chain residue and the diameter of the scleroglucan trimer rod were found to be $0.30 \pm 0.03$ and $2.6 \pm 0.5 \mathrm{~nm}$, respectively, which agree with the reported pitch (per residue) and diameter of the schizophyllan triple helix. Thus, it was concluded that the scleroglucan trimer has essentially the same triple helical structure as that of the schizophyllan trimer. Evidence was obtained showing that the higher aggregates in $0.01 \mathrm{~N} \mathrm{NaOH}$ consist of trimers as building units.
\end{abstract}

KEY WORDS Polysaccharide / Scleroglucan / Schizophyllan / Triple Helix / Radius of Gyration / Intrinsic Viscosity / Sedimentation Coefficient /

Scleroglucan is the general name for capsular polysaccharides elaborated by species of the genus Sclerotium. Recently, we ${ }^{1}$ showed that one of these polysaccharides, commercially available under the trade name of Polytran (Ceca S. A., France), is a $\beta$ 1,3-D-glucan consisting of the same repeating units as those of schizophyllan, ${ }^{2,3}$ an extracellular polysaccharide produced by a fungus Schizophyllum commune. We also found that Polytran is dissolved as a rodlike trimer in $0.01 \mathrm{~N}$ aqueous sodium hydroxide $(\mathrm{NaOH})$ and as a single randomly coiled chain in dimethylsulfoxide (DMSO), as is schizophyllan $^{4,5}$ in these solvents. In fact, these two glucans in the former solvent showed no discernible difference with respect to dimensional and viscosity behavior. However, their random coils in the latter solvent were distinctly different in the degree of swelling by excluded-volume effect. Actually, Polytran's coil appeared to be in the unperturbed state. It is not easy to reconcile this difference with the finding that Polytran and schizophyllan are chemically indistinguishable.

The point is that our experiment was done only on two Polytran samples. It seemed mandatory for a definitive elucidation of Polytran's random coil in DMSO to repeat a similar experiment with more samples covering a wide range of molecular weight. We therefore prepared 11 such Polytran samples and carried out light scattering, viscosity, and sedimentation velocity measurements both in $0.01 \mathrm{~N}$ $\mathrm{NaOH}$ and in DMSO. This paper presents the experimental data obtained and their theoretical interpretation.

\section{EXPERIMENTAL}

\section{Samples}

Eight scleroglucan samples different in molecular weight were prepared by sonicating a purified sample $^{1}$ of Polytran R. Each was separated into four to seven parts by fractional precipitation with water as the solvent and acetone as the precipitant. 
Appropriate middle fractions, designated below as H-1, F-1, D-3, I-1, I-3, C-2, C-5, B-3, J-1, J-3, and $\mathrm{E}-4$, were selected, reprecipitated from aqueous solutions into acetone, and freeze-dried from aqueous solutions. These fractions were dried overnight in vacuo before use.

\section{Light Scattering}

Intensities of light scattered from $0.01 \mathrm{~N} \mathrm{NaOH}$ and DMSO solutions of scleroglucan at $25^{\circ} \mathrm{C}$ were measured on a Fica 50 automatic light scattering photometer in an angular range from 22.5 to $150^{\circ}$. Vertically polarized incident light of 436 or $546 \mathrm{~nm}$ wavelength was used. The instrument was calibrated with benzene at $25^{\circ} \mathrm{C}$ as the reference liquid. The Rayleigh ratios of $25^{\circ} \mathrm{C}$ benzene at 436 and $546 \mathrm{~nm}$ were taken to be $46.5 \times 10^{-6}$ and $16.1 \times 10^{-6} \mathrm{~cm}$, respectively. ${ }^{6}$ The depolarization ratio of benzene was estimated to be $0.41_{0}$ for $436 \mathrm{~nm}$ and $0.40_{6}$ for $546 \mathrm{~nm}$ by the method of Rubingh and $\mathrm{Yu} .{ }^{7}$ For the specific refractive index increments of scleroglucan in $0.01 \mathrm{~N} \mathrm{NaOH}$ and DMSO, the previously determined values ${ }^{1}$ were used.

DMSO solutions were optically clarified by centrifugation at about $4 \times 10^{4}$ gravities for $2 \mathrm{~h}$, after being allowed to stand at $25-50^{\circ} \mathrm{C}$ for $16-52 \mathrm{~h}$. For $0.01 N \mathrm{NaOH}$ solutions, optical clarification was made as described by Kashiwagi et al. ${ }^{5}$

Analysis of the data for samples I-1, C-2, and E-4 by the method described elsewhere ${ }^{8}$ indicated that the optical anisotropy of scleroglucan in $0.01 \mathrm{~N}$ $\mathrm{NaOH}$ is negligible, as found by Kashiwagi et al. ${ }^{5}$ and Sato et $a l .{ }^{9}$ for schizophyllan in the same solvent.

\section{Ultracentrifugation}

Samples B-3 and E-4 in water at $25^{\circ} \mathrm{C}$ were investigated by sedimentation equilibrium, using a Spinco Model E ultracentrifuge equipped with an electronic speed-control system. A Kel-F $12 \mathrm{~mm}$ double-sector cell was used. The liquid column was adjusted to $1.4-1.6 \mathrm{~mm}$, and rotor speeds were chosen as 5200 and $7200 \mathrm{rpm}$ for samples B-3 and E-4, respectively. The partial specific volume of scleroglucan in water at $25^{\circ} \mathrm{C}$ was determined to be $0.620 \mathrm{~cm}^{3} \mathrm{~g}^{-1}$.

Sedimentation velocities of samples C-2, C-5, B3, J-1, J-3, and E-4 in $0.01 N \mathrm{NaOH}$ at $25^{\circ} \mathrm{C}$ were measured at a rotor speed of $48000 \mathrm{rpm}$, using a
Kel-F $30 \mathrm{~mm}$ single-sector cell. For any of these samples, the observed schlieren patterns were singlepeaked, so that sedimentation coefficients $s$ for a series of initial polymer mass concentrations $c_{0}$ were determined by the usual peak method.

\section{Viscometry}

Zero shear-rate viscosities of samples $\mathrm{H}-1, \mathrm{~F}-1$, D-3, I-1, and I-3 in $0.01 N \mathrm{NaOH}$ at $25^{\circ} \mathrm{C}$ were determined using four-bulb capillary viscometers. For other samples in $0.01 \mathrm{~N} \mathrm{NaOH}$ and all the samples in DMSO at $25^{\circ} \mathrm{C}$, conventional capillary viscometers of the Ubbelohde type were used.

\section{RESULTS}

\section{Molecular Weight}

Figure 1 illustrates the concentration dependence of $K c / R_{0}$ for samples I-3, C-5, and J-3 in $0.01 N$ $\mathrm{NaOH}$ and DMSO. Here, $K$ is the optical constant, $c$, the polymer mass concentration, and $R_{0}$, the reduced scattering intensity at zero scattering angle. It can be seen that the data points for each sample in $0.01 \mathrm{~N} \mathrm{NaOH}$ follow a straight line, whereas those in DMSO follow a curve bending upward. Since similar curvatures were observed for the concentration dependence of $K c / R_{0}$ for all other samples in DMSO, the $K c / R_{0}$ data in this solvent were anal-

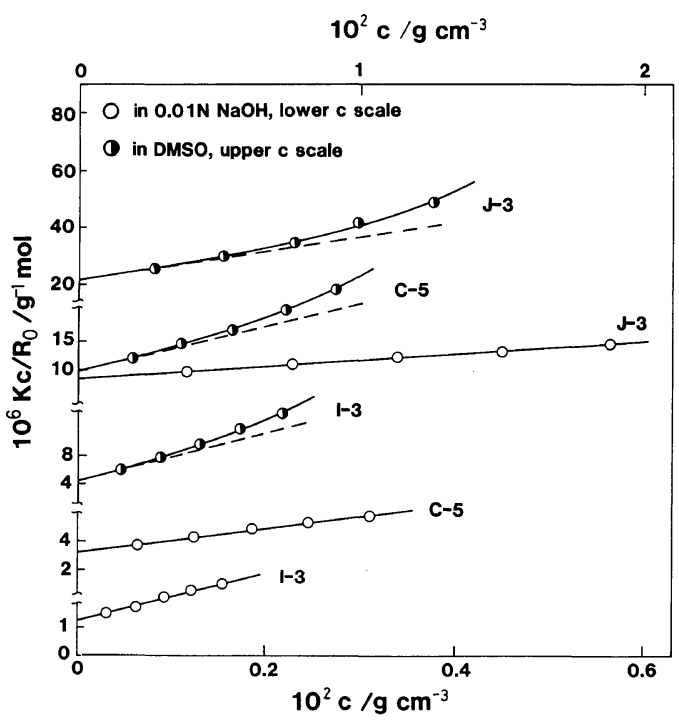

Figure 1. Plots of $K c / R_{0} v s . c$ for scleroglucan samples in $0.01 \mathrm{~N} \mathrm{NaOH}$ (the lower $c$ scale) and DMSO (the upper $c$ scale) at $25^{\circ} \mathrm{C}$. 
yzed using the Berry square-root plot. ${ }^{10}$

The values of $M_{w}$ (the weight-average molecular weight) and $A_{2}$ (the second virial coefficient) for all the scleroglucan samples in $0.01 N \mathrm{NaOH}$ and DMSO are summarized in Table $I$, along with those of $M_{w}$ and $M_{z} / M_{w}\left(M_{z}\right.$ is the $z$-average molecular weight) in pure water determined by sedimentation equilibrium. The $M_{z} / M_{w}$ values for samples B-3 and E-4 indicate that these two samples are narrow in molecular weight dispersion.

The values of $M_{w}(0.01 N \mathrm{NaOH}) / M_{w}$ (DMSO) presented in Table I indicate that the predominant species of scleroglucan in $0.01 \mathrm{~N} \mathrm{NaOH}$ is a trimer for $M_{w}$ (DMSO) below $2 \times 10^{5}$ and an aggregate higher than a trimer for $M_{w}$ (DMSO) above $2 \times 10^{5}$ and that the number of chains in the aggregate increases with an increase in $M_{w}$ (DMSO). This was an unexpected finding, since our previous determination $^{5}$ of $M_{w}(0.01 N \mathrm{NaOH}) / M_{w}$ (DMSO) for schizophyllan exhibited no significant deviation from 3 throughout the entire range of $M_{w}$ (DMSO) treated, i.e., $3.7 \times 10^{4}-1.6 \times 10^{6}$.

\section{Radius of Gyration}

Figure 2 depicts the particle scattering function $P(\theta)$ for scleroglucan samples in $0.01 N \mathrm{NaOH}$. The values of $\left\langle S^{2}\right\rangle^{1 / 2}$ (the radius of gyration) evaluated from the slopes of the indicated dashed lines are presented in Table I, along with those in DMSO. In Figure 3 , these and previously reported $\left\langle S^{2}\right\rangle^{1 / 2}$ values $^{1}$ in $0.01 N \mathrm{NaOH}$ and DMSO are compared with the data (the solid lines) of Kashiwagi et al. ${ }^{5}$ for schizophyllan in the same solvents.

All the data points for scleroglucan in $0.01 \mathrm{~N}$ $\mathrm{NaOH}$ fall close to the solid line for schizophyllan in the same solvent. The slope of this line equals 1.0 for $M_{w}(0.01 \mathrm{~N} \mathrm{NaOH})$ below $3 \times 10^{5}$ and decreases gradually with increasing $M_{w}(0.01 N \mathrm{NaOH})$. Thus, it may be concluded that the scleroglucan trimer $\left[M_{w}(0.01 N \mathrm{NaOH})<8 \times 10^{5}\right]$ is almost rigidly rodlike below and semiflexible above $M_{w}(0.01 N$ $\mathrm{NaOH}) \sim 3 \times 10^{5}$. However, the finding that $\left\langle S^{2}\right\rangle^{1 / 2}$ of both scleroglucan and schizophyllan in $0.01 \mathrm{~N}$ $\mathrm{NaOH}$ follow the same line throughout the entire range of $M_{w}(0.01 \mathrm{~N} \mathrm{NaOH})$ must be considered accidental, since, as has been mentioned above, the predominant species of these glucans in $0.01 \mathrm{~N}$ $\mathrm{NaOH}$ are different aggregates when $M_{w}$ (DMSO) is higher than about $2 \times 10^{5}$.

In Figure 3, except for the two highest-molecular
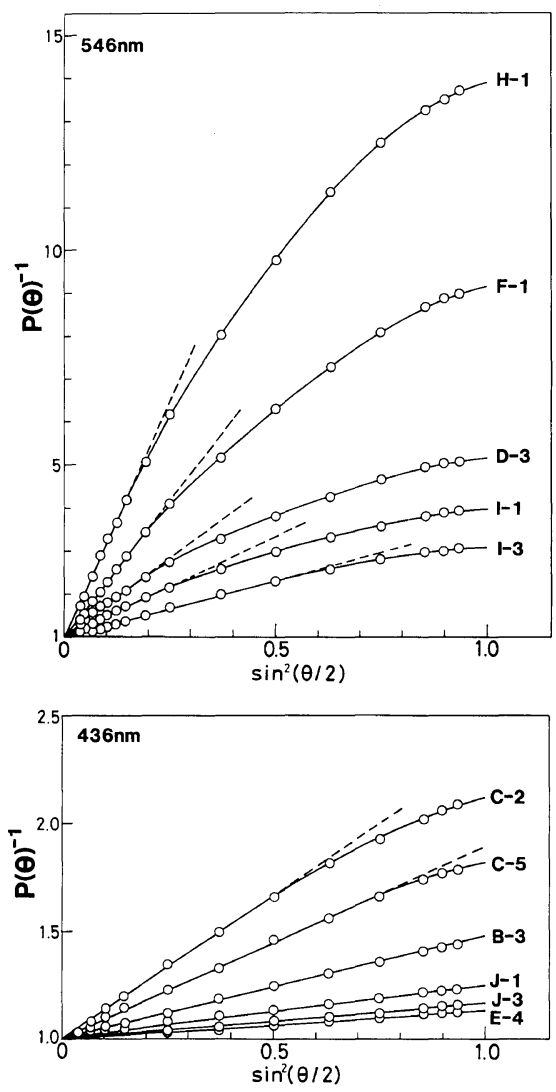

Figure 2. Particle scattering function for scleroglucan samples in $0.01 \mathrm{~N} \mathrm{NaOH}$ at $25^{\circ} \mathrm{C}$.

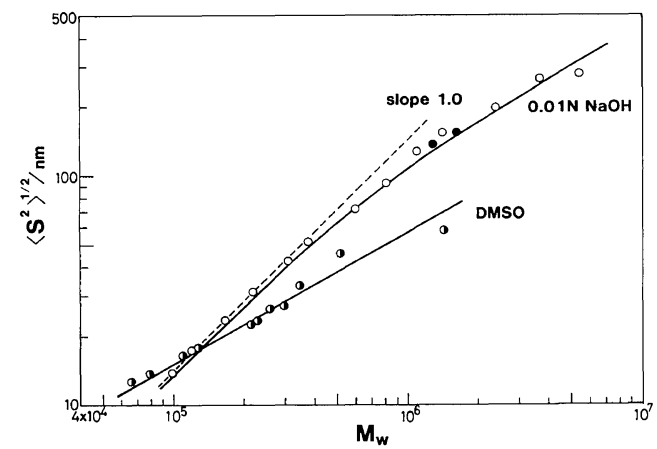

Figure 3. Comparison of $\left\langle S^{2}\right\rangle^{1 / 2}$ data for scleroglucan in $0.01 N \mathrm{NaOH}(\mathrm{O})$ and DMSO $(O)$ with those (the solid lines) for schizophyllan ${ }^{5}$ in the same solvents. The filled circles represent the data for scleroglucan samples $\mathrm{H}-1$ and $\mathrm{F}-1$ in $0.05 \mathrm{~N} \mathrm{NaOH}$.

weight samples, $\left\langle S^{2}\right\rangle^{1 / 2}$ of scleroglucan in DMSO falls on a straight line of slope 0.58 , fitting schizophyllan $\left\langle S^{2}\right\rangle^{1 / 2}$ in the same solvent. This indicates 
that, in DMSO, the scleroglucan random coil is perturbed by the excluded-volume effect as much as the schizophyllan random coil, and thus contradicts our previous conclusion ${ }^{1}$ that Polytran's coil in DMSO is virtually in the unperturbed state. The present $A_{2}$ values for DMSO solutions (Table I)

Table I. Results from light scattering measurements on scleroglucan samples in $0.01 \mathrm{~N} \mathrm{NaOH}$ and DMSO at $25^{\circ} \mathrm{C}$

\begin{tabular}{|c|c|c|c|c|c|c|c|c|}
\hline \multirow{4}{*}{ Sample } & \multicolumn{4}{|c|}{$0.01 N \mathrm{NaOH}$} & \multicolumn{3}{|c|}{ DMSO } & \multirow{4}{*}{$\frac{M_{w}(0.01 N \mathrm{NaOH})}{M_{w}(\mathrm{DMSO})}$} \\
\hline & \multirow{3}{*}{$10^{-4} M_{w}$} & \multirow{2}{*}{$10^{4} A_{2}$} & \multirow{2}{*}{$\left\langle S^{2}\right\rangle^{1 / 2}$} & \multirow{3}{*}{$\frac{M_{z}}{M_{w}}$} & \multirow{3}{*}{$10^{-4} M_{w}$} & \multirow{3}{*}{$\frac{10^{4} A_{2}}{\mathrm{~cm}^{3} \mathrm{~mol} \mathrm{~g}^{-2}}$} & \multirow{3}{*}{$\frac{\left\langle S^{2}\right\rangle^{1 / 2}}{n m}$} & \\
\hline & & & & & & & & \\
\hline & & $\mathrm{cm}^{3} \mathrm{~mol} \mathrm{~g}^{-2}$ & $\mathrm{~nm}$ & & & & & \\
\hline \multirow[t]{2}{*}{$\mathrm{H}-1$} & 370 & 2.8 & 264 & & 51.8 & 3.9 & 46.0 & 7.1 \\
\hline & $163^{\mathrm{a}}$ & $2.4^{\mathrm{a}}$ & $151^{\mathrm{a}}$ & & & & & \\
\hline \multirow{2}{*}{ F-1 } & 239 & 5.4 & 198 & & 37.2 & 5.3 & 33.1 & 6.4 \\
\hline & $129^{\mathrm{a}}$ & $3.2^{\mathrm{a}}$ & $134^{\mathrm{a}}$ & & & & & \\
\hline D-3 & 141 & 4.4 & 152 & & 29.5 & 4.3 & 27.1 & 4.8 \\
\hline $\mathrm{I}-1$ & 110 & 1.7 & 127 & & 25.8 & 4.9 & 26.4 & 4.3 \\
\hline $\mathrm{I}-3$ & 81.1 & 4.1 & 92.7 & & 22.5 & 4.7 & 23.2 & 3.6 \\
\hline $\mathrm{C}-2$ & 37.6 & 5.7 & 51.4 & & 12.8 & 6.8 & 17.8 & 2.9 \\
\hline C-5 & 31.0 & 4.3 & 42.1 & & 11.0 & 6.3 & 16.3 & 2.8 \\
\hline \multirow[t]{2}{*}{ B-3 } & 22.0 & 6.2 & 31.1 & & 7.89 & 6.5 & 13.8 & 2.8 \\
\hline & $21.2^{\mathrm{b}}$ & $1.5^{\mathrm{b}}$ & & $1.1_{2}^{\mathrm{b}}$ & & & & \\
\hline $\mathrm{J}-1$ & 16.7 & 8.0 & 23.3 & & 6.57 & 5.5 & 12.6 & 2.5 \\
\hline $\mathrm{J}-3$ & 12.0 & 5.8 & 17.4 & & 4.68 & 8.3 & & 2.6 \\
\hline \multirow[t]{2}{*}{ E-4 } & 9.62 & 7.8 & 13.4 & & 3.56 & 6.7 & & 2.7 \\
\hline & $9.01^{\mathrm{b}}$ & $3.2^{\mathrm{b}}$ & & $1.1_{9}^{\mathrm{b}}$ & & & & \\
\hline
\end{tabular}

a In $0.05 \mathrm{~N} \mathrm{NaOH}$.

b Sedimentation equilibrium in water.

Table II. Results from viscosity and sedimentation velocity measurements on scleroglucan samples in $0.01 \mathrm{~N} \mathrm{NaOH}$ and DMSO at $25^{\circ} \mathrm{C}$

\begin{tabular}{|c|c|c|c|c|c|c|}
\hline \multirow{3}{*}{ Sample } & \multicolumn{4}{|c|}{$0.01 N \mathrm{NaOH}$} & \multicolumn{2}{|c|}{ DMSO } \\
\hline & $10^{-2}[\eta]$ & & $10^{13} s_{0}$ & $k_{\mathrm{s}}$ & $10^{-2}[\eta]$ & \\
\hline & $\mathrm{cm}^{3} \mathrm{~g}^{-1}$ & & $\mathrm{~s}$ & $\mathrm{~cm}^{3} \mathrm{~g}^{-1}$ & $\mathrm{~cm}^{3} \mathrm{~g}^{-1}$ & \\
\hline \multirow[t]{2}{*}{$\mathrm{H}-1$} & 43.7 & 0.45 & & & 2.17 & 0.32 \\
\hline & $25.3^{\mathrm{a}}$ & $0.44^{\mathrm{a}}$ & & & & \\
\hline \multirow[t]{2}{*}{ F-1 } & 26.1 & 0.43 & & & 1.80 & 0.34 \\
\hline & $17.0^{\mathrm{a}}$ & $0.44^{\mathrm{a}}$ & & & & \\
\hline D-3 & 14.2 & 0.42 & & & 1.39 & 0.34 \\
\hline $\mathrm{I}-1$ & 10.5 & 0.43 & & & 1.26 & 0.36 \\
\hline $\mathrm{I}-3$ & 6.59 & 0.41 & & & 1.09 & 0.34 \\
\hline $\mathrm{C}-2$ & 2.65 & 0.41 & 7.42 & 75 & 0.781 & 0.35 \\
\hline C-5 & 2.03 & 0.42 & 7.17 & 64 & 0.740 & 0.33 \\
\hline B-3 & 1.34 & 0.42 & 6.80 & 62 & 0.562 & 0.38 \\
\hline $\mathrm{J}-1$ & 0.809 & 0.42 & 6.29 & 53 & 0.462 & 0.35 \\
\hline$J-3$ & 0.497 & 0.43 & 5.73 & 42 & 0.376 & 0.40 \\
\hline E-4 & 0.345 & 0.44 & 5.34 & 34 & 0.305 & 0.42 \\
\hline
\end{tabular}

${ }^{\text {a }}$ In $0.05 \mathrm{~N} \mathrm{NaOH}$. 


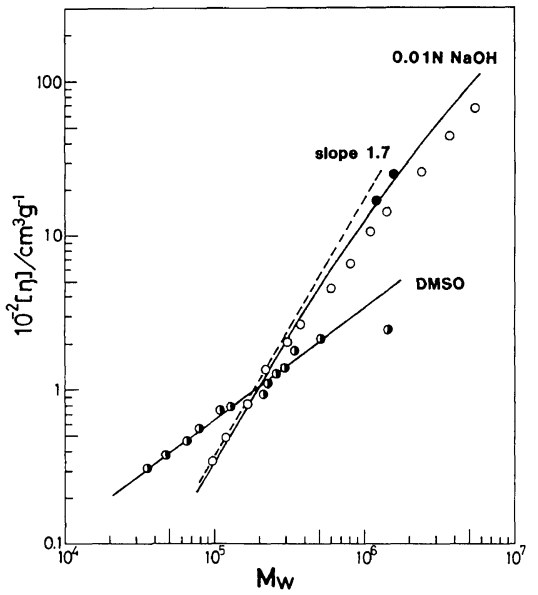

Figure 4. Comparison of $[\eta]$ data for scleroglucan in $0.01 N \mathrm{NaOH}(\mathrm{O})$ and DMSO (O) with those (the solid lines) for schizophyllan ${ }^{4,5}$ in the same solvents. The filled circles represent the data for scleroglucan samples $\mathrm{H}-1$ and $\mathrm{F}-1$ in $0.05 \mathrm{~N} \mathrm{NaOH}$.

support this finding, being much larger than those previously obtained and consistent with random coils perturbed by excluded-volume effect. Thus, it appears convincing that our previous conclusion was incorrect. But what is responsible for this?

We suspect that the previous test solutions might have contained a small amount of aggregates since they were prepared at room temperature. We therefore heated DMSO solutions of our previous Polytran samples at $50^{\circ} \mathrm{C}$ for $48 \mathrm{~h}$ and then measured their light scattering intensities. However, the results were substantially the same as before, giving $A_{2} \sim 0$. At present, it is not clear as to why the two scleroglucan samples chosen in our previous study behave in DMSO so differently from those investigated in the present work.

\section{Intrinsic Viscosity and Sedimentation Coefficient}

Numerical data for $[\eta]$ (the intrinsic viscosity) and $k^{\prime}$ (Huggins' constant) in $0.01 \mathrm{~N} \mathrm{NaOH}$ and DMSO are summarized in Table II. These and previous data $^{1}$ of $[\eta]$ are compared with the corresponding data (the solid lines) for schizophyllan ${ }^{4,5}$ in Figure 4. The present data for $M_{w}$ below $3 \times 10^{5}$ in $0.01 N$ $\mathrm{NaOH}$ can be fitted by a straight line of slope 1.7 (the dashed line), which almost merges with the solid line for this solvent and is consistent with the finding from $\left\langle S^{2}\right\rangle$ that scleroglucan trimers with $M_{w}(0.01 N \mathrm{NaOH})$ lower than $3 \times 10^{5}$ are rigidly

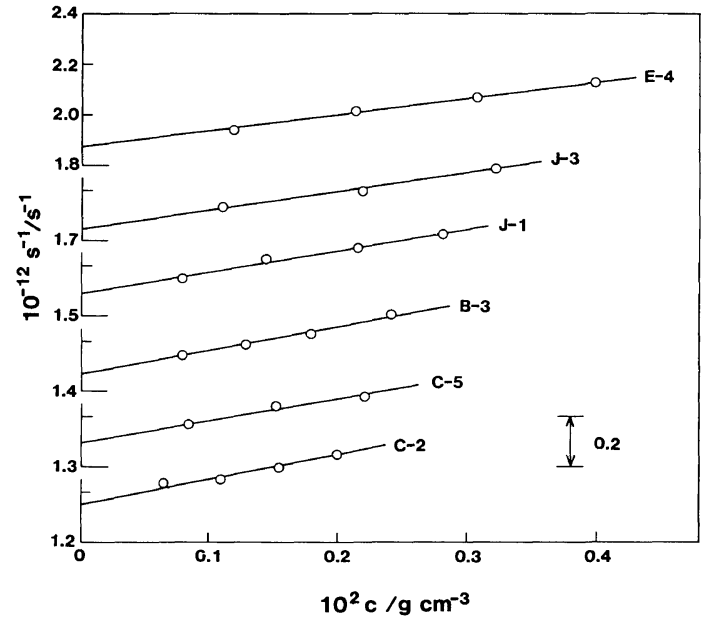

Figure 5. Concentration dependence of $s^{-1}$ for scleroglucan samples in $0.01 \mathrm{~N} \mathrm{NaOH}$ at $25^{\circ} \mathrm{C}$.

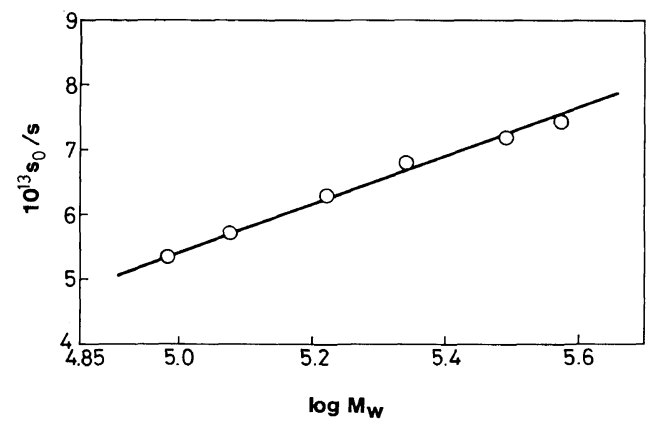

Figure 6. Plot of $s_{0}$ vs. $\log M_{w}$ for scleroglucan in $0.01 N \mathrm{NaOH}$.

rodlike. On the other hand, except for the highestmolecular weight sample, the DMSO data fall on the schizophyllan line of slope 0.69 , indicating that both scleroglucan and schizophyllan single chains in this solvent are in the random coil conformation perturbed by volume effect.

Figure 5 shows the concentration dependence of $s^{-1}$ in $0.01 N \mathrm{NaOH}$. The values of $s_{0}$ (the limiting sedimentation coefficient) and $k_{\mathrm{s}}$ (the constant defined by the equation $s^{-1}=s_{0}{ }^{-1}+k_{\mathrm{s}} s_{0}{ }^{-1} c_{0}$ ) determined from the indicated straight lines are presented in the fourth and fifth columns of Table II. Figure 6 shows that these $s_{0}$ vary linearly with log $M_{w}$. This linear relation is additional evidence for the fact that the scleroglucan trimer is rigidly rodlike. 


\section{DISCUSSION}

\section{Data Analysis}

Our dimensional and hydrodynamic data along with those of the molecular weight ratio $M_{w}(0.01 \mathrm{~N}$ $\mathrm{NaOH}) / M_{w}$ (DMSO) show that scleroglucan dissolves in $0.01 \mathrm{~N} \mathrm{NaOH}$ as a rigid trimer for $M_{w}$ (DMSO) lower than $1 \times 10^{5}$. The well-known expression for $\left\langle S^{2}\right\rangle$ of a rigid rod is

$$
\left\langle S^{2}\right\rangle=M /\left(12 M_{\mathrm{L}}\right)
$$

where $M$ is the molecular weight of the polymer and $M_{\mathrm{L}}$, the molar mass per unit length of the rod. If our $\left\langle S^{2}\right\rangle$ data for $M_{w}$ (DMSO) below $1 \times 10^{5}$ in $0.01 N$ $\mathrm{NaOH}$ are substituted into this equation, $M_{\mathrm{L}}$ is found to be $2050 \pm 50 \mathrm{~nm}^{-1}$.

Yamakawa's expressions ${ }^{11,12}$ for $[\eta]$ and $s_{0}$ of a long straight cylinder are

$$
\begin{aligned}
& M^{2} /[\eta]=\left(45 M_{\mathrm{L}}^{3} / 2 \pi N_{\mathrm{A}}\right) \\
& {\left[\ln M-0.6970-\ln \left(d M_{\mathrm{L}}\right)\right]} \\
& s_{0}=\left[\left(1-\bar{v} \rho_{0}\right) M_{\mathrm{L}} / 3 \pi \eta_{0} N_{\mathrm{A}}\right] \\
& {\left[\ln M+0.3863-\ln \left(d M_{\mathrm{L}}\right)\right]}
\end{aligned}
$$

where $d$ is the diameter of the cylinder, $N_{\mathrm{A}}$ Avogadro's constant, $\bar{v}$ the partial specific volume of the polymer, and $\rho_{0}$ and $\eta_{0}$ the solvent density and viscosity, respectively. The values of $M_{w}{ }^{2} /[\eta]$ calculated from our data for the four lowestmolecular weight samples in $0.01 \mathrm{~N} \mathrm{NaOH}$ are plotted against $\ln M_{w}$ in Figure 7. If a straight line is drawn to fit the plotted points as indicated and the values of its slope and intercept at $\ln M_{w}=0$ are substituted into eq 2, $M_{\mathrm{L}}$ and $d$ are found to be $2200 \pm 60 \mathrm{~nm}^{-1}$ and $2.6 \pm 0.4 \mathrm{~nm}$, respectively. Further, when compared with eq 3 , the linear re- lation between $s_{0}$ and $\log M_{w}$ in Figure 6 yields $2140 \pm 70 \mathrm{~nm}^{-1}$ for $M_{\mathrm{L}}$ and $2.5 \pm 0.4 \mathrm{~nm}$ for $d$.

\section{Triple Helical Structure}

The repeating unit of scleroglucan contains three main chain glucose residues (see Figure 1 of ref 1). Hence, the length $h$ per main chain residue of the scleroglucan trimer along the rod axis can be written

$$
h=\left(M_{0} / 3\right) /\left(M_{\mathrm{L}} / 3\right)
$$

with $M_{0}$ the molar mass of the scleroglucan repeating unit $\left(=648 \mathrm{~g} \mathrm{~mol}^{-1}\right)$. The values of $h$ calculated from our $M_{\mathrm{L}}$ and those of $d$ estimated above are summarized in Table III, along with the pitches and diameters for the schizophyllan triple helix in water ${ }^{13}$ and $0.01 \mathrm{~N} \mathrm{NaOH}{ }^{5}$ and the model triple helix ${ }^{4}$ of schizophyllan. Our $h$ values estimated by different methods agree with one another and also with the schizophyllan helix pitches. Our $d$ values are also in substantial agreement with

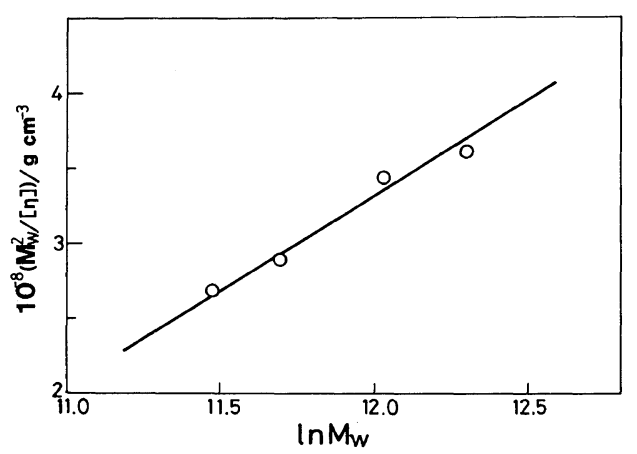

Figure 7. Plot of $M_{w}{ }^{2} /[\eta] v s$. $\ln M_{w}$ for scleroglucan in $0.01 \mathrm{~N} \mathrm{NaOH}$.

Table III. Pitches and diameters of the triple helices of scleroglucan and schizophyllan

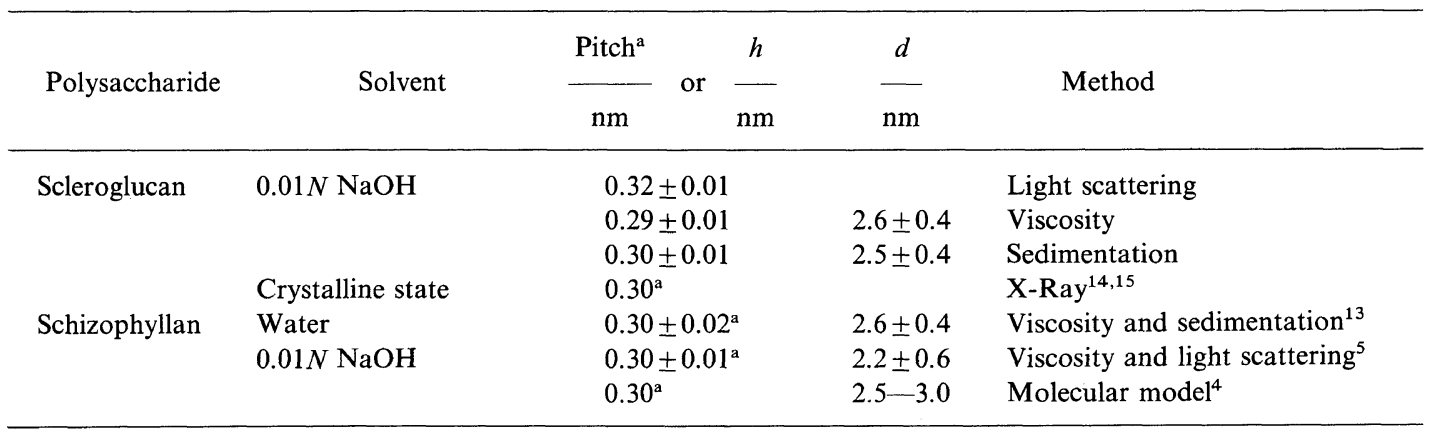

\footnotetext{
a Per residue.
} 
those for schizophyllan and its model helix. Thus, it may be concluded that the scleroglucan trimer has a triple helical structure very similar to that of schizophyllan.

Very recently, Marchessault et al. ${ }^{14,15}$ concluded from X-ray and conformational studies that scleroglucan in the crystalline state has a triple helical structure with a pitch (per residue) of $0.30 \mathrm{~nm}$, which can be favorably compared with the values from our solution study. Thus, it may be concluded that the triple helical structure of scleroglucan in the crystalline state is maintained in dilute aqueous $\mathrm{NaOH}$.

\section{Aggregates of Triple Helices}

We have mentioned that our scleroglucan samples with $M_{w}$ (DMSO) higher than $2 \times 10^{5}$ are dispersed in $0.01 \mathrm{~N} \mathrm{NaOH}$ as aggregates higher than a trimer. In the following, we present some convincing data that these higher aggregates are built up of two or more trimers.

According to Kashiwagi et al., ${ }^{5}$ the addition of $\mathrm{NaOH}$ to an aqueous solution of schizophyllan enhances the solubility of the triple helix, but leads to both dissociation (to single chains) and gradual degradation of the helix when the $\mathrm{NaOH}$ concentration $x$ exceeds $0.1 \mathrm{~N}$. We suspected that this was probably the case with scleroglucan, and tried to check it by measuring the viscosity of highermolecular weight samples $\mathrm{H}-1$ and $\mathrm{F}-1$ in aqueous $\mathrm{NaOH}$ of different $x$. For comparison, we also investigated lower-molecular weight samples B-3 and $\mathrm{J}-1$, which dissolve as trimers in $0.01 \mathrm{~N} \mathrm{NaOH}$.

The results are illustrated in Figure 8. It can be seen that $[\eta]$ for samples B-3 and J-1 are constant up to $x \sim 0.05 N$, decrease sharply at $x \sim 0.1 N$, and level off at constant values above $x \sim 0.2 N$. The abrupt change in $[\eta]$ at $x \sim 0.1 N$ is probably a reflection of the dissociation of the triple helix to single chains, since the leveling-off values of $[\eta]$ for $x$ above $0.2 \mathrm{~N}$ are comparable to $[\eta]$ of the respective samples in DMSO. The curves for samples $\mathrm{H}-1$ and F-1 comprise two distinct steps, each showing a shoulder around $x=0.05 N$. Probably, the decline to the shoulder represents the dissociation of a higher aggregate to trimers, and the subsequent sharp decline, the breaking of trimers into single chains.

In order to confirm this expectation, light scattering and the viscosity of samples $\mathrm{H}-1$ and F-1 in $0.05 \mathrm{~N} \mathrm{NaOH}$ were measured. The specific refrac-

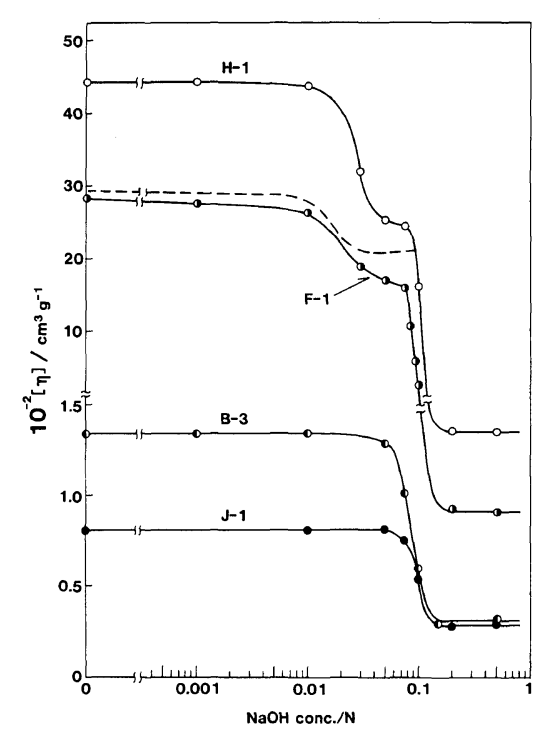

Figure 8. Dependence of $[\eta]$ on the concentration of $\mathrm{NaOH}$ for scleroglucan samples in aqueous $\mathrm{NaOH}$ at $25^{\circ} \mathrm{C}$. The dashed line indicates the data of Bluhm et al. $^{15}$

tive index increment in this solvent at either 436 or $546 \mathrm{~nm}$ wavelength was not different from that in $0.01 \mathrm{~N} \mathrm{NaOH}$. The data obtained are given in Tables I and II and shown by filled circles in Figures 3 and 4 . It can be seen that these circles lie close to the solid lines for schizophyllan in $0.01 \mathrm{~N}$ $\mathrm{NaOH}$ and also give $M_{w}$ values approximately 3 times as large as those in DMSO. This demonstrates that the aggregates of samples $\mathrm{H}-1$ and F-1 are dissociated in $0.05 \mathrm{~N} \mathrm{NaOH}$ to trimers which have the same structure as the schizophyllan triple helix.

Recently, Bluhm et al. ${ }^{15}$ investigated the $\mathrm{pH}$ dependence of the reduced viscosity $\eta_{\mathrm{sp}} / c \quad(c=$ $4.9 \times 10^{-4} \mathrm{~g} \mathrm{~cm}^{-3}$ ) of a Polytran sample in $8 \times 10^{-2} \mathrm{M}$ aqueous sodium chloride containing $\mathrm{NaOH}$ (for $\mathrm{pH}>7$ ) or hydrochloric acid (for $\mathrm{pH}<7$ ). Their experimental data are shown by a dashed line in Figure 8 after $\mathrm{pH}$ has been converted to $x$ assuming the relation $\log x=\mathrm{pH}-14$. Bluhm et al. remarked that the values of $\eta_{\mathrm{sp}} / c$ for $x$ above $0.03 N$ are comparable to the reported $[\eta]$ for schizophyllan in DMSO, and attributed a fairly sharp decrease in $\eta_{\mathrm{sp}} / c$ at $x \sim 0.015 \mathrm{~N}$ to the dissociation of triple helices to random coils. However, their remark on $\eta_{\mathrm{sp}} / c$ for $x$ above $0.03 N$ is incorrect, since such $\eta_{\mathrm{sp}} / c$ values far exceed the high- 
est of $[\eta]\left(4.65 \times 10^{2} \mathrm{~cm}^{3} \mathrm{~g}^{-1}\right)$ ever obtained for schizophyllan in DMSO. ${ }^{4}$ More reasonably, the sharp decrease in $\eta_{\mathrm{sp}} / c$ at $x \sim 0.015 N$ may be explained as duc to the dissociation of higher aggregates to triple helices.

\section{CONCLUDING REMARKS}

The present study has shown that the trimer of Polytran scleroglucan in dilute aqueous $\mathrm{NaOH}$ and its monomer in DMSO assume essentially the same conformations as those of schizophyllan in these solvents. This finding is compatible with the results from a chemical analysis ${ }^{1}$ and $\mathrm{C}^{13}$-NMR measurements ${ }^{16}$ that the two glucans have the same chemical structure. However, these glucans in $0.01 \mathrm{~N}$ $\mathrm{NaOH}$ differed in solubility behavior when $M_{w}$ (DMSO) was higher than $2 \times 10^{5}$. In this region, triple helices of schizophyllan remained intact, while those of scleroglucan tended to associate further with one another to higher aggregates. This difference is striking, but we do not know the reason for it. Native schizophyllan produced by Schizophyllum commune in a culture medium separates spontaneously from the mycelium and migrates freely into the liquid phase. ${ }^{17}$ On the other hand, scleroglucan produced by Sclerotium rolfsii adheres to the mycelium as a gellike aggregate and cannot be dispersed in the liquid phase unless the culture medium is heated and homogenized. ${ }^{18}$ Such a difference in the native states of these two glucans may bear some relation to the difference in their solubility in $0.01 \mathrm{~N} \mathrm{NaOH}$.

Acknowledgment. We are very grateful to Professor H. Fujita for his advice, interest, and comments on this manuscript.

\section{REFERENCES}

1. T. Yanaki, T. Kojima, and T. Norisuye, Polym. J., 13, 1135 (1981).

2. S. Kikumoto, T. Miyajima, K. Kimura, S. Okubo, and N. Komatsu, J. Agric. Chem. Soc. Jpn., 45, 162 (1971).

3. K. Tabata, W. Ito, T. Kojima, S. Kawabata, and A. Misaki, Carbohydr. Res., 89, 121 (1981).

4. T. Norisuye, T. Yanaki, and H. Fujita, J. Polym. Sci., Polym. Phys. Ed., 18, 547 (1980).

5. Y. Kashiwagi, T. Norisuye, and H. Fujita, Macromolecules, 14, 1220 (1981).

6. Gj. Deželić and J. Vavra, Croat. Chem. Acta, 38, 35 (1966).

7. D. N. Rubingh and H. Yu, Macromolecules, 9, 681 (1976).

8. H. Murakami, T. Norisuye, and H. Fujita, Macromolecules, 13, 345 (1980).

9. T. Sato, T. Norisuye, and H. Fujita, Macromolecules, 16, 185 (1983).

10. G. C. Berry, J. Chem. Phys., 44, 4550 (1966).

11. H. Yamakawa, Macromolecules, 8, 339 (1975).

12. H. Yamakawa and M. Fujii, Macromolecules, 6, 407 (1973).

13. T. Yanaki, T. Norisuye, and H. Fujita, Macromolecules, 13, 1462 (1980).

14. R. H. Marchessault, Y. Deslandes, T. L. Bluhm, H. Chanzy, and A. Sarko, "XIth International Carbohydrate Symposium," Vancouver, 1982, Abstract, V-24.

15. T. L. Bluhm, Y. Deslandes, R. H. Marchessault, S. Pérez, and M. Rinaudo, Carbohydr. Res., 100, 117 (1982).

16. M. Rinaudo and M. Vincendon, Carbohydr. Polym., 2, 135 (1982).

17. S. Kikumoto, T. Miyajima, S. Yoshizumi, S. Fujimoto, and K. Kimura, J. Agric. Chem. Soc. Jpn., 44, 337 (1970).

18. Ceca S. A. Technical Report, Nov., 1976. 